\title{
DDF and Pohlmeyer invariants of (super)string
}

\author{
Urs Schreiber \\ Universität Duisburg-Essen \\ Essen, 45117, Germany \\ E-mail: Urs.Schreiber@uni-essen.de
}

\begin{abstract}
We show how the Pohlmeyer invariants of the bosonic string are expressible in terms of DDF invariants. Quantization of the DDF observables in the usual way yields a consistent quantization of the algebra of Pohlmeyer invariants. Furthermore it becomes straightforward to generalize the Pohlmeyer invariants to the superstring as well as to all backgrounds which allow a free field realization of the worldsheet theory.
\end{abstract}




\section{Contents}

1. Introduction 2

2. DDF operators and Pohlmeyer invariants

2.1 DDF operators

2.2 Pohlmeyer invariants

2.3 Classical bosonic DDF invariants and their relation to the Pohlmeyer invariants

2.3.1 Classical bosonic DDF invariants

2.3.2 Expressing Pohlmeyer invartiants in terms of DDF invariants

2.3.3 DDF and Pohlmeyer invariants for superstring 14

3. Summary and Conclusion 15 


\section{Introduction}

The classical string is known to be a completely integrable system with an infinite number of classical observables that Poisson-commute with all the constraints. A concise and comprehensive review of the work by Pohlmeyer, Rehren, Bahns, et. al. [1, 2, 3, 4] on a particular manifestation of these gauge invariant observables, known as Pohlmeyer invariants, is given in [5].

Since the Virasoro algebra is the direct sum of two copies of $\operatorname{diff}\left(S^{1}\right)$, the diffeomorphism algebra of the circle, invariant observables are simply those that are reparameterization invariant with respect to these two algebras. Two common types of reparameterization invariant objects are

- Wilson lines,

- integrals over densities of unit reparameterization weight .

More precisely (see $\$ 2.2$ (p.7) for a detailed derivation), let $\mathcal{P}_{ \pm}^{\mu}(\sigma)$ be the left- and rightmoving classical fields on the free closed bosonic string with Poisson brackets of the form

$$
\left[\mathcal{P}_{ \pm}^{\mu}(\sigma), \mathcal{P}_{ \pm}^{\nu}\left(\sigma^{\prime}\right)\right]_{\mathrm{PB}}= \pm \eta^{\mu \nu} \delta^{\prime}\left(\sigma, \sigma^{\prime}\right)
$$

These transform with unit weight under the action of the Virasoro algebra and hence the Wilson line

$$
\operatorname{Tr} \mathbf{P} \exp \left(\int_{S^{1}} \mathcal{P}_{+}^{\mu} A_{+\mu}\right) \operatorname{Tr} \mathbf{P} \exp \left(\int_{S^{1}} \mathcal{P}_{-}^{\mu} A_{-\mu}\right)
$$

Poisson-commutes with all Virasoro constraints (where $\mathbf{P}$ denotes path-ordering and $A_{ \pm \mu}$ are two constant Lie-algebra-valued 1-forms on target space). It is easy to see that also the coefficients of $\operatorname{Tr}\left(A^{n}\right)$ in the Taylor expansion of this object commute with the constraints. These coefficients are known as the Pohlmeyer invariants. The Poisson algebra of these observables is rather convoluted. The problem of finding a quantum deformation of this algebra turns out to be difficult and involved and has up to now remained unsolved [2, 5, 6]. Furthermore, by itself, it is not obvious how the above construction should generalize to the superstring.

For these reasons it seems worthwhile to consider the possibility of alternatively using integrals over unit weight densities to construct a complete set of classical invariant charges. A little reflection shows that the well-known DDF operators [7] for the covariantly quantized string, which are operators that commute with all the quantum (super-)Virasoro constraints, are built using essentially this principle:

From elementary CFT it follows that for $\mathcal{O}(z)$ any primary CFT field of conformal weight $h=1$ we have (after the usual introduction of a complex coordinate $z$ on the worldsheet)

$$
\left[L_{n}, \oint d z \mathcal{O}(z)\right]=0, \quad \forall n
$$


By choosing $\mathcal{O}(z)=\left[G_{-\nu}, \tilde{\mathcal{O}}(z)\right]$, (with $G_{-\nu}$ the $(\nu=0)$-mode (R sector) or $(\nu=-1 / 2)$ mode (NS sector) of the worldsheet supercurrent and $h(\tilde{\mathcal{O}})=1 / 2$ ) this generalizes to the superstring

$$
\left[G_{n-\nu}, \oint d z \mathcal{O}(z)\right]=0, \quad \forall n
$$

as is reviewed below in $\$ 2.1$. It hence only remains to find $\mathcal{O}$ (or $\tilde{\mathcal{O}}$ ) of weight 1 (or $1 / 2)$ such that the resulting integrals have nice (super-)commutators and exhaust the space of all invariant charges. Doing this in a natural way yields the DDF operators.

It is readily checked that this construction of the DDF operators can be mimicked in terms of the classical Poisson algebra to yield a complete set of classical invariants which we shall call classical DDF invariants. These inherit all the nice properties of their quantum cousins.

Most importantly, as is shown in $\S 2.3$ (p.9), the Pohlmeyer invariants can be expressed in terms of the classical DDF invariants. Since it is known how the latter have to be quantized (i.e. the crucial quantum corrections to these charges is well known $c f$. $\$ 2.1$ (p.⿴囗十) this also tells us how the Pohlmeyer invariants can consistently be quantized.

In particular this shows that any normal ordering in the quantization of the Pohlmeyer invariants must be applied only inside each DDF operator, while the DDF operators among themselves need not be reordered. This clarifies the result of [6], where it was demonstrated that the Pohlmeyer invariants cannot be consistently quantized by writing them in terms of worldsheet oscillators and applying normal ordering with respect to these. Rather, as will be shown here, one has to replace these oscillators with the corresponding DDF observables, and the assertion is that the Pohlmeyer invariants, like any other reparameterization invariant observable, are unaffected by this replacement.

Because the DDF operators, together with the identity operator, form a closed algebra, the quantization of the Pohlmeyer invariants in terms of DDF operators, as demonstrated here, is manifestly consistent in the sense that the quantum commutator of two such invariants is itself again an invariant.

It should be emphasized that, in contrast to what has been stated in [6], the construction of classical DDF invariants does not require that any worldsheet coordinate gauge has to be fixed, in particular their construction has nothing to do with fixing conformal gauge. This is obvious due to the fact that the classical DDF invariants are constructable (as the Pohlmeyer invariants, too) by proceeding from just the Nambu-Goto action, which does not even have an auxiliary worldsheet metric which could be gauge fixed. Furthermore the canonical data and the form of the Virasoro constraints as obtained from the Nambu-Goto action are precisely the same as those obtained from the Polyakov action with or without fixed worldsheet gauge.

Furthermore, our proof that the Pohlmeyer invariants can be equivalently expressed in terms of DDF invariants (i.e. certain polynomials in DDF invariants are equal to the Pohlmeyer invariants) constructively demonstrates that both are on the same footing as far as requirements for their respective construction is concerned. 
The organization of this paper is as follows:

We first review the construction of DDF operators in $\$ 2.1$ (p. 1 ) and then that of Pohlmeyer invariants in $\$ 2.2$ (p.7).

Then in $\$ 2.3 .1$ (p.9) we discuss the classical DDF invariants in detail, show how they can be used to express the Pohlmeyer invariants (2.3.2 (p.13)) and how this generalizes to the superstring $(\S 2.3 .3$ (p.14) $)$.

Summary and discussion is given in $\$ 3(\mathrm{p} .15)$.

A brief summary of the results presented here will be published in [8].

\section{DDF operators and Pohlmeyer invariants}

We review first the DDF operators, then the Pohlmeyer invariants, then show how both are related.

\subsection{DDF operators}

The construction of DDF operators [7] is very well known, but to the best of our knowledge there is no comprehensive review of all possible cases (transversal and longitudinal, bosonic

and fermionic) available in the standard literature. The following section tries to list and derive all the essential facts.

In the standard textbook literature one can find

- in [9] (in non-CFT language) the construction of

- transversal bosonic (§2.3.2)

- transversal supersymmetric (§4.3.2)

- longitudinal bosonic (pp. 111),

- and in [10] (in CFT language) the construction of

- transversal bosonic (eq. (8.2.29))

DDF states, which go back to [7].

The following summarizes and derives (in CFT language) all

- transversal and longitudinal

- bosonic and fermionic

DDF operators (for a free supersymmetric worldsheet theory).

Using the standard normalization of the OPE

$$
\begin{aligned}
X^{\mu}(z) X^{\nu}(0) & \sim-\frac{\alpha^{\prime}}{2} \eta^{\mu \nu} \ln z \\
\psi^{\mu}(z) \psi^{\nu}(0) & \sim \frac{\eta^{\mu \nu}}{z}
\end{aligned}
$$


for the bosonic and fermionic worldsheet fields, the (super-)Virasoro currents read

$$
\begin{aligned}
T(z) & =-\frac{1}{\alpha^{\prime}} \partial X \cdot \partial X(z)-\frac{1}{2} \psi \cdot \partial \psi \\
T_{\mathrm{F}}(z) & =i \sqrt{\frac{\alpha^{\prime}}{2}} \psi \cdot \partial X .
\end{aligned}
$$

The DDF operators are defined as a set of operators that commute with all modes of $T$ and $T_{\mathrm{F}}$ (are gauge invariant observables) and satisfy an algebra that mimics that of worldsheet oscillator creation/annihilation operators.

First of all one needs to single out two linearly independent lightlike Killing vectors $l$ and $k$ on target space, and in the context of this subsection we choose to normalize them as $l \cdot k=2$. The span of $l$ and $k$ is called the longitudinal space and its orthogonal complement is the tranverse space.

For $\mathcal{O}(z)=\sum_{-\infty}^{\infty} \mathcal{O}_{m} z^{-(m+h)}$ a primary field of weight $h$ we shall refer to the OPE $T(z) \mathcal{O}(0) \sim \frac{h}{z^{2}} \mathcal{O}(0)+\frac{1}{z} \partial \mathcal{O}(0)$ as the tensor law in some of the following formulas, instead of writing out all terms. The modes of $T$ and $T_{\mathrm{F}}$ are denoted by $L_{m}$ and $G_{m-\nu}$ as usual.

The elementary but crucial fact used for the construction of DDF operators is that 0-modes of tensor operators of weight $h=1$ commute with all $L_{m}$ generators according to

$$
\left[L_{m}, \mathcal{O}_{n}\right]=((h-1) m-n) \mathcal{O}_{m+n}
$$

Therefore the task of finding DDF states is reduced to that of finding linearly independent $h=1$ fields that have the desired commutation relations and, in the case of the superstring, are closed with respect to $T_{\mathrm{F}}$ (see below).

Bosonic string. For the bosonic string the DDF operators $A_{n}^{\mu}$ are defined by

$$
A_{n}^{\mu} \propto \oint \frac{d z}{2 \pi i}\left(\partial X^{\mu}+k^{\mu} \frac{\alpha^{\prime}}{8} i n \partial \ln (k \cdot \partial X)\right) e^{i n k X}(z) .
$$

(These are of course nothing but integrated vertex operators of the massless fields. Note that the logarithmic terms of $k \cdot \partial X$, as well as the inverse powers that will be used further below, are well defined operators, as is discussed above equation (2.3.87) in [9].)

It is straightforward to check that the operators (2.4) are really invariant:

First consider the transverse DDF operators. For $v$ a transverse target space vector (such that in particular $v \cdot k=0$ ) the operator $v \cdot A_{n}$ is manifestly the 0-mode of an $h=1$ primary field (the exponential factor has $h=0$ due to $k \cdot k=0$ ) and hence is invariant.

Furthermore $k \cdot A_{n} \propto \delta_{n, 0} k \cdot \oint \partial X$ (for $n \neq 0$ the integrand is a total derivative) also obvioulsy commutes with the $L_{m}$.

The only subtlety arises for the longitudinal $l \cdot A_{n}$. Here, the non-tensor behaviour of

$$
T(z) l \cdot \partial X e^{i n k X}(w) \sim-\frac{\alpha^{\prime}}{2} \frac{i n}{(z-w)^{3}} e^{i n k X}(w)+(h=1) \text {-tensor law }
$$


is precisely canceled by the curious logarithmic correction term $\partial \ln (k \cdot \partial X)(z)=\frac{k \partial^{2} X}{k \partial X}(z)$. Namely because of

$$
T(z) \partial^{2} X^{\mu}(w) \sim \frac{2 \partial X^{\mu}(w)}{(z-w)^{3}}+(h=2) \text {-tensor law }
$$

one has

$$
\Rightarrow T(z) \frac{k \cdot \partial^{2} X}{k \cdot \partial X} e^{i n k X}(w) \sim \frac{2 e^{i n k X}}{(z-w)^{3}}+(h=1) \text {-tensor law },
$$

which hence makes the entire integrand of $l \cdot A_{m}$ transform as an $h=1$ primary, as desired.

Superstring. The analogous construction for the superstring has to ensure in addition that the DDF operators commute with the supercharges $G_{m-\nu}$. This is simply achieved by 'closing' the integral over a given weight $h=1 / 2$ primary field $D(z)$ to obtain the operator

$$
\left[G_{-\nu}, D_{\nu}\right]_{\iota}=\left[\oint \frac{d z}{2 \pi i} T_{F}(z), \oint \frac{d z}{2 \pi i} D(z)\right] \quad\left\{\begin{array}{ll}
\nu=0 & \text { R sector } \\
\nu=1 / 2 & \text { NS sector }
\end{array} .\right.
$$

Here and in the remainder of this subsection the brackets denote supercommutators.

The resulting operator is manifestly the zero mode of a weight $h=1$ tensor and hence commutes with all $L_{n}$. Furthermore it commutes with $G_{-\nu}$ because of

$$
\left[G_{-\nu},\left[G_{-\nu}, D_{\nu}\right]_{\iota}\right]_{\iota}=\left[L_{-2 \nu}, D_{\nu}\right]_{\iota} \stackrel{(2.3)}{=} 0 .
$$

Since $G_{-\nu}$ and $L_{m}, \forall m$ generate the entire algebra, the 'closed' operator $\left[G_{-\nu}, D_{\nu}\right]$ indeed commutes with all $L_{m}$ and $G_{m-\nu}, \forall m$.

It is therefore clear that the superstring DDF operators, which can be defined as

$$
\begin{aligned}
A_{n}^{\mu} & :=\left[G_{\nu}, \oint \frac{d z}{2 \pi i} \psi^{\mu} e^{i n k X}(z)\right] \\
B_{n}^{\mu} & :=\left[G_{\nu}, \oint \frac{d z}{2 \pi i}\left(\psi^{\mu} k \cdot \psi-\frac{1}{4} k^{\mu} \partial \ln (k \cdot \partial X)\right) \frac{e^{i n k X}}{\sqrt{k \cdot \partial X}}\right]
\end{aligned}
$$

commute with the super-Virasoro generators, since the second arguments of the commutators are integrals over weight $1 / 2$ tensors. (And of course the latter are nothing but the integrated vertex operators as they appear in the (-1) superghost picture). The nature and purpose of the logarithmic correction term in the second line is just as discussed for the bosonic theory above: It cancels the non-tensor term in

$$
T(z) l \cdot \psi k \cdot \psi \frac{e^{i k X}}{\sqrt{k \cdot \partial X}}(w) \sim \frac{1}{(z-w)^{3}} \frac{e^{i n k X}}{\sqrt{k \cdot \partial X}}+(h=1 / 2) \text {-tensor law } .
$$

Evaluating the above supercommutators yields the explicit form for $A_{n}^{\mu}$ and $B_{n}^{\mu}$ :

$$
\begin{aligned}
A_{n}^{\mu}= & i \sqrt{\frac{2}{\alpha^{\prime}}} \oint \frac{d z}{2 \pi i}\left(\partial X^{\mu}+\frac{\alpha^{\prime}}{2} i n \psi^{\mu} k \cdot \psi\right) e^{i n k X}(z) \\
B_{n}^{\mu}= & i \sqrt{\frac{2}{\alpha^{\prime}}} \oint \frac{d z}{2 \pi i}\left(\partial X^{\mu} k \cdot \psi-\psi^{\mu} k \cdot \partial X+\frac{\alpha^{\prime}}{4} \psi^{\mu} k \cdot \psi k \cdot \partial \psi \frac{1}{k \cdot \partial X}\right) \frac{e^{i n k X}}{\sqrt{k \cdot \partial X}}(z) \\
& +i \sqrt{\frac{2}{\alpha^{\prime}}} \oint \frac{d z}{2 \pi i} k^{\mu}\left(k \cdot \psi f_{1}(k \cdot X, k \cdot \partial X)+k \cdot \partial \psi f_{2}(k \cdot X, k \cdot \partial X)\right)(z),
\end{aligned}
$$


where $f_{1}$ and $f_{2}$ are functions which we don not need to write out here.

The above discussion has focused on only a single chirality sector (left-moving, say). It must be noted that the exponent $i n k \cdot X$ involved in the definition of all the above DDF operators contains the 0 -mode $k \cdot x$ of the coordinate field $k \cdot X$. The existence of this 0 mode implies that the above DDF operators do not commute with the (super-)Virasoro generators of the opposite chirality. In order to account for that one has to suitably multiply left- and right-moving DDF operators. The details of this will be discussed in $\$ 2.3 .1$ (p.99).

\subsection{Pohlmeyer invariants}

We now turn to the classical bosonic string and discuss the invartiants which have been studied by Pohlmeyer et al.

In the literature the invariance of the Pohlmeyer charges is demonstrated by the method of Lax pairs. But the same fact follows already from the well-known reparameterization invariance property of Wilson loops. To recall how this works for the classical bosonic string consider the following:

Denote the left- or rightmoving classical worldsheet fields in canonical language by $\mathcal{P}^{\mu}(\sigma)$, which have the canonical Poisson bracket

$$
\left[\mathcal{P}^{\mu}(\sigma), \mathcal{P}^{\nu}\left(\sigma^{\prime}\right)\right]_{\mathrm{PB}}=-\eta^{\mu \nu} \delta^{\prime}\left(\sigma-\sigma^{\prime}\right) .
$$

The modes of the Virasoro constraints are

$$
L_{m}:=\frac{1}{2} \int d \sigma e^{-i m \sigma} \eta_{\mu \nu} \mathcal{P}^{\mu}(\sigma) \mathcal{P}^{\nu}(\sigma)
$$

and the $\mathcal{P}(\sigma)$ transform with unit weight under their Poisson action:

$$
\left[L_{m}, \mathcal{P}^{\mu}(\sigma)\right]_{\mathrm{PB}}=\left(e^{-i m \sigma} \mathcal{P}^{\mu}(\sigma)\right)^{\prime} .
$$

This is all one needs to show that the Pohlmeyer invariants $Z^{\mu_{1} \cdots \mu_{N}}$ defined by

$$
Z^{\mu_{1} \cdots \mu_{N}}(\mathcal{P}):=\frac{1}{N} \int_{0}^{2 \pi} d \sigma^{1} \int_{\sigma^{1}}^{\sigma^{1}+2 \pi} d \sigma^{2} \cdots \int_{\sigma^{N-1}}^{\sigma^{1}+2 \pi} d \sigma^{N} \mathcal{P}^{\mu_{1}}\left(\sigma^{1}\right) \mathcal{P}^{\mu_{2}}\left(\sigma^{2}\right) \cdots \mathcal{P}^{\mu_{N}}\left(\sigma^{N}\right)
$$

Poisson-commute with all the $L_{m}$.

The proof involves just a little combinatorics and algebra:

First note that if $F\left(\sigma^{1}, \sigma^{2}, \cdots, \sigma^{N}\right)$ is any function which is periodic with period $2 \pi$ in each of its $N$ arguments, the cyclically permuted path-ordered integral over $F$ is equal to the integral used in (2.16)

$$
\begin{aligned}
& {\left[\underset{0<\sigma^{1}<\sigma^{2}<\cdots<\sigma^{N}<2 \pi}{\int d^{N} \sigma}+\underset{0<\sigma^{N}<\sigma^{1}<\cdots<\sigma^{N-1}<2 \pi}{\int} d^{N} \sigma+\int_{0<\sigma^{N-1}<\sigma^{N}<\cdots<\sigma^{N-2}<2 \pi} d^{N} \sigma\right] F\left(\sigma_{1}, \sigma_{2}, \cdots, \sigma_{N}\right)} \\
& =\int_{0}^{2 \pi} d \sigma^{1} \int_{\sigma^{1}}^{\sigma^{1}+2 \pi} d \sigma^{2} \cdots \int_{\sigma^{N-1}}^{\sigma^{1}+2 \pi} d \sigma^{N} F\left(\sigma_{1}, \sigma_{2}, \cdots, \sigma_{N}\right) \text {. }
\end{aligned}
$$


(This follows by noting that while, for instance, $\sigma^{1}$ runs from 0 to $2 \pi$ all other $\sigma^{i}$ can be taken to run from $\sigma^{1}$ to $\sigma^{1}+2 \pi$ while remaining in the correct order.)

This shows that the Pohlmeyer observables (2.16) are invariant under cyclic permutation of their indices. It can also be used to write their variation as

$$
\begin{gathered}
\delta Z^{\mu_{1} \cdots \mu_{N}}=\frac{1}{N} \int_{0}^{2 \pi} d \sigma^{1} \int_{\sigma^{1}}^{\sigma^{1}+2 \pi} d \sigma^{2} \cdots \int_{\sigma^{N-1}}^{\sigma^{1}+2 \pi} d \sigma^{N}\left(\mathcal{P}^{\mu_{1}}\left(\sigma^{1}\right) \mathcal{P}^{\mu_{2}}\left(\sigma^{2}\right) \cdots \delta \mathcal{P}^{\mu_{N}}\left(\sigma^{N}\right)+\right. \\
+\mathcal{P}^{\mu_{N}}\left(\sigma^{1}\right) \mathcal{P}^{\mu_{1}}\left(\sigma^{2}\right) \cdots \delta \mathcal{P}^{\mu_{N-1}}\left(\sigma^{N}\right)+ \\
+\cdots)
\end{gathered}
$$

because we may cyclically permute the integration variables. But if one now sets $\delta \mathcal{P}^{\mu}(\sigma)=$ $\left[L_{m}, \mathcal{P}^{\mu}(\sigma)\right]_{\mathrm{PB}}$ one gets, using (2.15),

$$
\begin{aligned}
& \delta Z^{\mu_{1} \cdots \mu_{N}}= \\
& \frac{1}{N} \int_{0}^{2 \pi} d \sigma^{1} \int_{\sigma^{1}}^{\sigma^{1}+2 \pi} d \sigma^{2} \cdots \int_{\sigma_{N-2}}^{\sigma^{1}+2 \pi} d \sigma^{N-1}( \\
& \quad \xi \mathcal{P}^{\mu_{N}} \mathcal{P}^{\mu_{1}}\left(\sigma^{1}\right) \cdots \mathcal{P}^{\mu_{N-1}}\left(\sigma^{N-1}\right)-\mathcal{P}^{\mu_{1}}\left(\sigma^{1}\right) \cdots \xi \mathcal{P}^{\mu_{N-1}} \mathcal{P}^{\mu_{N}}\left(\sigma^{N-1}\right)+ \\
& \quad+\xi \mathcal{P}^{\mu_{N-1}} \mathcal{P}^{\mu_{N}}\left(\sigma^{1}\right) \cdots \mathcal{P}^{\mu_{N-2}}\left(\sigma^{N-1}\right)-\mathcal{P}^{\mu_{N}}\left(\sigma^{1}\right) \cdots \xi \mathcal{P}^{\mu_{N-2}} \mathcal{P}^{\mu_{N-1}}\left(\sigma^{N-1}\right) \\
& \quad+\cdots \\
& \quad \cdots
\end{aligned}
$$

(where we have written $\xi(\sigma)=e^{-i m \sigma}$ for brevity). The contributions from the innermost integration cancel due to the cyclic permutation of integrands and integration variables.

We note that the identity $\left[L_{m}, Z^{\mu_{1} \cdots \mu_{N}}(\mathcal{P})\right]_{\mathrm{PB}}=0$ is just the infinitesimal version of the fact that the Pohlmeyer observables are invariant under finite reparameterizations

$$
\mathcal{P}(\sigma) \mapsto \tilde{\mathcal{P}}(\sigma):=R^{\prime}(\sigma) \mathcal{P}(R(\sigma))
$$

induced by the invertible function $R$ which is assumed to satisfy

$$
R(\sigma+2 \pi)=R(\sigma)+2 \pi
$$

Indeed, we have the important relation

$$
Z^{\mu_{1} \cdots \mu_{N}}(\mathcal{P})=Z^{\mu_{1} \cdots \mu_{N}}(\tilde{\mathcal{P}})
$$

which is at the heart of our derivation in $\$ 2.3 .2$ (p.13) that the Pohlmeyer invariants can be expressed in terms of DDF invariants 
The proof of this involves just a simple change of variables in the integral:

$$
\begin{aligned}
& Z^{\mu_{1} \cdots \mu_{N}}(\tilde{\mathcal{P}}) \\
& =\frac{1}{N} \int_{0}^{2 \pi} d \sigma^{1} \int_{\sigma^{1}}^{\sigma^{1}+2 \pi} d \sigma^{2} \ldots \int_{\sigma^{N-1}}^{\sigma^{1}+2 \pi} d \sigma^{N} R^{\prime}\left(\sigma^{1}\right) R^{\prime}\left(\sigma^{2}\right) \cdots R^{\prime}\left(\sigma^{N}\right) \mathcal{P}^{\mu_{1}}\left(R\left(\sigma^{1}\right)\right) \cdots \mathcal{P}^{\mu_{N}}\left(R\left(\sigma^{N}\right)\right) \\
& \stackrel{\tilde{\sigma}^{i}:=R\left(\sigma^{i}\right)}{=} \frac{1}{N} \int_{R(0)}^{R(2 \pi)} d \tilde{\sigma}^{1} \int_{R\left(\sigma^{1}\right)}^{R\left(\sigma^{1}+2 \pi\right)} d \tilde{\sigma}^{2} \ldots \int_{R\left(\sigma^{N-1}\right)}^{R\left(\sigma^{1}+2 \pi\right)} d \tilde{\sigma}^{N} \mathcal{P}^{\mu_{1}}\left(\tilde{\sigma}^{1}\right) \mathcal{P}^{\mu_{2}}\left(\tilde{\sigma}^{2}\right) \cdots \mathcal{P}^{\mu_{N}}\left(\tilde{\sigma}^{N}\right) \\
& \stackrel{2.21)}{=} \frac{1}{N} \int_{R(0)}^{R(0)+2 \pi} d \tilde{\sigma}^{1} \int_{\tilde{\sigma}^{1}}^{\tilde{\sigma}^{1}+2 \pi} d \tilde{\sigma}^{2} \ldots \int_{\tilde{\sigma}^{N-1}}^{\tilde{\sigma}^{1}+2 \pi} d \tilde{\sigma}^{N} \mathcal{P}^{\mu_{1}}\left(\tilde{\sigma}^{1}\right) \mathcal{P}^{\mu_{2}}\left(\tilde{\sigma}^{2}\right) \cdots \mathcal{P}^{\mu_{N}}\left(\tilde{\sigma}^{N}\right) \\
& =Z^{\mu_{1} \cdots \mu_{N}}(\mathcal{P}) .
\end{aligned}
$$

Finally, for the sake of completeness, we note the well-known fact that the Pohlmeyer invariants appear naturally as the Taylor-coefficients of Wilson loops along the string at constant worldsheet time. Let $A_{\mu}$ be a constant but otherwise arbitrary $\operatorname{GL}(N, \mathbb{C})$ connection on target space, then the Wilson loop around the string of this connection with respect to $\mathcal{P}$ is

$$
\operatorname{Tr} \mathbf{P} \exp \left(\int_{0}^{2 \pi} d \sigma A_{\mu} \mathcal{P}^{\mu}(\sigma)\right)=\sum_{n=0}^{\infty} Z^{\mu_{1} \cdots \mu_{n}}(\mathcal{P}) \operatorname{Tr}\left(A_{\mu_{1}} \cdots A_{\mu_{2}}\right),
$$

where $\mathbf{P}$ denotes path-ordering.

This way of getting string "states" by means of Wilson lines of constant (but possibly large $N$ ) gauge connections along the string is intriguingly reminiscent of similar constructions used in the IIB Matrix Model (IKKT model) 11.

In the next sections the classical DDF invariants are described and it is shown how the Pohlmeyer invariants can be expressed in terms of these.

\subsection{Classical bosonic DDF invariants and their relation to the Pohlmeyer in- variants}

The construction of classical DDF-like invariants for the superstring, which is the content of $\$ 2.3 .3$ (p.14), is straightforward once the bosonic case is understood. The basic idea is very simple and shall therefore be given here first for the bosonic string, in order to demonstrate how $\$ 2.1$ (p.4) and $\$ 2.2$ (p. (7) fit together.

\subsubsection{Classical bosonic DDF invariants}

In order to establish our notation and sign conventions we briefly list some definitions and relations which are in principle well known from elementary CFT but are rarely written out in the canonical language which we will need here. 
So let $X(\sigma)$ and $P(\sigma)$ be canonical coordinates and momenta of the bosonic string with Poisson brackets

$$
\left[X^{\mu}(\sigma), P_{\nu}(\kappa)\right]_{\mathrm{PB}}=\delta_{\nu}^{\mu} \delta(\sigma-\kappa) .
$$

In close analogy to the CFT notation $\partial X$ and $\bar{\partial} X$ we define

$$
\mathcal{P}_{ \pm}^{\mu}(\sigma)=\frac{1}{\sqrt{2 T}}\left(P^{\mu}(\sigma) \pm T X^{\prime \mu}(\sigma)\right)
$$

(Here $T=1 / 2 \pi \alpha^{\prime}$ is the string tension and we assume a trivial Minkowski background and shift all spacetime indices with $\eta_{\mu \nu}=\operatorname{diag}(-1,1, \cdots, 1)$.)

Their Poisson brackets are of course

$$
\begin{aligned}
& {\left[\mathcal{P}_{ \pm}^{\mu}(\sigma), \mathcal{P}_{ \pm}^{\nu}(\kappa)\right]_{\mathrm{PB}}= \pm \eta^{\mu \nu} \delta^{\prime}(\sigma-\kappa)} \\
& {\left[\mathcal{P}_{ \pm}^{\mu}(\sigma), \mathcal{P}_{\mp}^{\nu}(\kappa)\right]_{\mathrm{PB}}=0 .}
\end{aligned}
$$

From the mode expansion

$$
\begin{aligned}
& \mathcal{P}_{+}^{\mu}(\sigma):=\frac{1}{\sqrt{2 \pi}} \sum_{m} \tilde{\alpha}_{m}^{\mu} e^{-i m \sigma} \\
& \mathcal{P}_{-}^{\mu}(\sigma):=\frac{1}{\sqrt{2 \pi}} \sum_{m} \alpha_{m}^{\mu} e^{+i m \sigma}
\end{aligned}
$$

one finds the string oscillator Poisson algebra

$$
\left[\alpha_{m}^{\mu}, \alpha_{n}^{\nu}\right]_{\mathrm{PB}}=-i m \eta^{\mu \nu} \delta_{m+n, 0},
$$

as well as

$$
\left[x^{\mu}, p^{\nu}\right]_{\mathrm{PB}}=\eta^{\mu \nu},
$$

where

$$
\begin{aligned}
x^{\mu} & :=\frac{1}{2 \pi} \int X^{\mu}(\sigma) d \sigma \\
p^{\mu} & :=\int P^{\mu}(\sigma) d \sigma=\frac{1}{\sqrt{4 \pi T}} \alpha_{0}=\frac{1}{\sqrt{4 \pi T}} \tilde{\alpha}_{0} .
\end{aligned}
$$

In terms of these oscillators the field $X^{\prime}$ reads

$$
\begin{aligned}
X^{\prime \mu}(\sigma) & =\frac{1}{\sqrt{2 T}}\left(\mathcal{P}_{+}^{\mu}(\sigma)-\mathcal{P}_{-}^{\mu}(\sigma)\right) \\
& =\frac{1}{\sqrt{4 \pi T}} \sum_{m=-\infty}^{\infty}\left(-\alpha_{m}^{\mu}+\tilde{\alpha}_{-m}^{\mu}\right) e^{+i m \sigma}
\end{aligned}
$$

and hence the canonical coordinate field itself is

$$
X^{\mu}(\sigma)=x^{\mu}+\frac{i}{\sqrt{4 \pi T}} \sum_{m \neq 0} \frac{1}{m}\left(\alpha_{m}^{\mu}-\tilde{\alpha}_{-m}^{\mu}\right) e^{+i m \sigma} .
$$


Any field $A(\sigma)$ is said to have classical conformal weight $w(A)$ iff

$$
\left[L_{m}, A(\sigma)\right]=e^{-i m \sigma} A^{\prime}(\sigma)+w(A)\left(e^{-i m \sigma}\right)^{\prime} A(\sigma)
$$

and is said to have classical conformal weight $\tilde{w}(A)$ iff

$$
\left[\tilde{L}_{m}, A(\sigma)\right]=-e^{+i m \sigma} A^{\prime}(\sigma)-\tilde{w}(A)\left(e^{+i m \sigma}\right)^{\prime} A(\sigma),
$$

where

$$
\begin{aligned}
& L_{m}:=\frac{1}{2} \int e^{-i m \sigma} \mathcal{P}_{-}(\sigma) \cdot \mathcal{P}_{-}(\sigma)=\frac{1}{2} \sum_{k=-\infty}^{\infty} \alpha_{m-k} \cdot \alpha_{k} \\
& \tilde{L}_{m}:=\frac{1}{2} \int e^{+i m \sigma} \mathcal{P}_{+}(\sigma) \cdot \mathcal{P}_{+}(\sigma)=\frac{1}{2} \sum_{k=-\infty}^{\infty} \tilde{\alpha}_{m-k} \cdot \tilde{\alpha}_{k}
\end{aligned}
$$

are the usual modes of the Virasoro generators.

The parts of $X(\sigma)$ which have $w=0$ and $\tilde{w}=0$, respectively, are

$$
X_{-}^{\mu}(\sigma):=x^{\mu}-\frac{\sigma}{4 \pi T} p^{\mu}+\frac{i}{\sqrt{4 \pi T}} \sum_{m \neq 0} \frac{1}{m} \alpha_{m}^{\mu} e^{+i m \sigma}
$$

and

$$
X_{+}^{\mu}(\sigma):=x^{\mu}+\frac{\sigma}{4 \pi T} p^{\mu}+\frac{i}{\sqrt{4 \pi T}} \sum_{m \neq 0} \frac{1}{m} \tilde{\alpha}_{m}^{\mu} e^{-i m \sigma}
$$

This is checked by noticing the crucial property

$$
\begin{aligned}
& \left(X_{-}^{\mu}\right)^{\prime}(\sigma)=-\frac{1}{\sqrt{2 T}} \mathcal{P}_{-}^{\mu}(\sigma) \\
& \left(X_{+}^{\mu}\right)^{\prime}(\sigma)=\frac{1}{\sqrt{2 T}} \mathcal{P}_{+}^{\mu}(\sigma) .
\end{aligned}
$$

These weight 0 fields can now be used to construct "invariant oscillators", namely the classical DDF invariants:

To that end fix any lightlike vector field $k$ on target space and consider the fields

$$
R_{ \pm}(\sigma):= \pm \frac{4 \pi T}{k \cdot p} k \cdot X_{ \pm}(\sigma) .
$$

The prefactor is an invariant and chosen so that

$$
R_{ \pm}(\sigma+2 \pi)=R_{ \pm}(\sigma)+2 \pi
$$

Furthermore the derivative of $R_{ \pm}$is

$$
R_{ \pm}^{\prime}(\sigma)=\frac{2 \pi \sqrt{2 T}}{k \cdot p} k \cdot \mathcal{P}_{ \pm}(\sigma)
$$

It has been observed [12] that this derivative vanishes only on a subset of phase space of vanishing measure. This can be seen as follows: 
The classical Virasoro constraints $\mathcal{P}_{ \pm}^{2}=0$ say that $\mathcal{P}_{ \pm}(\sigma)$ is lightlike. Because $k$ is also lightlike this implies that $k \cdot \mathcal{P}_{ \pm}(\sigma)$ vanishes iff $\mathcal{P}_{ \pm}(\sigma)$ is parallel to $k$.

By writing $\mathcal{P}_{ \pm}=\mathcal{P}_{ \pm}^{0}\left[1, \mathcal{P}_{ \pm}^{i} / \mathcal{P}_{ \pm}^{0}\right]$ and noting that the spatial unit vector $e_{ \pm}^{i}(\sigma):=$ $\mathcal{P}_{ \pm}^{i} / \mathcal{P}_{ \pm}^{0}$ is of weight $w=0$ or $\tilde{w}=0$ (while it Poisson commutes with the respective opposite Virasoro algebra), and hence transforms under the action of the Virasoro generators (which includes time evolution) as $e_{ \pm}^{i}(\sigma) \rightarrow e_{ \pm}^{i}(\sigma+f(\sigma))$, one sees that this condition is satisfied for some $\sigma$ at some instance of time if and only if it is satisfied for some $\sigma$ at any given time. In other words the time evolution of the string traces out trajectories in phase space which either have $\mathcal{P}_{ \pm}$parallel to $k$ for some $\sigma$ at all times or never.

In summary this means that except on the subset of phase space (of vanishing measure) of those trajectories where there exists a $\sigma$ such that $k \cdot \mathcal{P}_{ \pm}(\sigma)=0$, the observables $R_{ \pm}(\sigma)$ define invertible reparameterizations of the interval $[0,2 \pi)$, as considered in (2.21).

The above fact will be crucial below for expressing the Pohlmeyer invariants in terms of DDF invariants. For later usage let us introduce the notation $\mathbf{P}_{k}$ for the total phase space minus that set of vanishing measure:

$$
\mathbf{P}_{k}:=\left\{(X(\sigma), P(\sigma))_{\sigma \in(0,2 \pi)} \mid k \cdot \mathcal{P}_{ \pm}(\sigma) \neq 0 \forall \sigma\right\} .
$$

Now the classical DDF observables $A_{m}^{\mu}$ and $\tilde{A}_{m}^{\mu}$ of the closed bosonic string are finally defined (adapting the construction of (2.4) but using slighly different normalizations) by

$$
\begin{aligned}
A_{m}^{\mu} & :=\frac{1}{\sqrt{2 \pi}} \int d \sigma \mathcal{P}_{-}^{\mu}(\sigma) e^{-i m R_{-}(\sigma)} \\
\tilde{A}_{m}^{\mu} & :=\frac{1}{\sqrt{2 \pi}} \int d \sigma \mathcal{P}_{+}^{\mu}(\sigma) e^{i m R_{+}(\sigma)} .
\end{aligned}
$$

Note that the construction principle of these objects is essentially the same as that of the ordinary oscillators (2.28) except that the parameterization of the string used here differs from one point in phase space to the other.

Being integrals over fields of total weight $w=1$ and $\tilde{w}=1$, respectively, the DDF observables obviously Poisson-commute with their associated half of the Virasoro generators:

$$
\begin{aligned}
{\left[L_{m}, A_{n}^{\mu}\right] } & =0 \\
{\left[\tilde{L}_{m}, \tilde{A}_{n}^{\mu}\right] } & =0 .
\end{aligned}
$$

But due to the coordinate 0 -mode $\frac{2 T}{k p} k \cdot x$ that enters the definition of $R_{ \pm}$, the mixed Poisson-brackets do not vanish. In order to construct invariants one therefore has to split off this 0-mode and define the truncated observables

$$
\begin{aligned}
& a_{m}^{\mu}:=A_{m}^{\mu} e^{-i m \frac{2 T}{k p} k x} \\
& \tilde{a}_{m}^{\mu}:=A_{m}^{\mu} e^{-i m \frac{2 T}{k p} k x} .
\end{aligned}
$$

These now obviously have vanishing mixed Poisson brackets:

$$
\begin{aligned}
{\left[L_{m}, \tilde{a}_{n}^{\mu}\right] } & =0 \\
{\left[\tilde{L}_{m}, a_{n}^{\mu}\right] } & =0 .
\end{aligned}
$$


Therefore classical DDF invariants which Poisson commute with all Virasoro constraints are obtained by forming products

$$
D_{\left\{m_{i}, \tilde{m}_{j}\right\}}:=a_{m_{1}}^{\mu_{1}} \cdots a_{m_{r}}^{\mu_{r}} \tilde{a}_{\tilde{n}_{1}}^{\nu_{1}} \cdots a_{\tilde{m}_{s}}^{\nu_{s}} e^{i N \frac{2 T}{k p} k x}
$$

which satisfy the level matching condition:

$$
\sum_{i} m_{i}=N=\sum_{j} \tilde{m}_{j}
$$

In order to see this explicitly write

$$
\begin{aligned}
& {\left[L_{n}, D_{\left\{m_{i}, \tilde{m}_{j}\right\}}\right]_{\mathrm{PB}}=\underbrace{\left[L_{n}, a_{m_{1}}^{\mu_{1}} \cdots a_{m_{r}}^{\mu_{r}} e^{i N \frac{2 T}{k p} k x}\right]_{\mathrm{PB}}}_{[2.4)_{0}} \tilde{a}_{\tilde{n}_{1}}^{\nu_{1}} \cdots a_{\tilde{m}_{s}}^{\nu_{s}}+} \\
& +a_{m_{1}}^{\mu_{1}} \cdots a_{m_{r}}^{\mu_{r}} e^{i N \frac{2 T}{k p} k x} \underbrace{\left[L_{n}, \tilde{a}_{\tilde{n}_{1}}^{\nu_{1}} \cdots a_{\tilde{m}_{s}}^{\nu_{s}}\right]_{\mathrm{PB}}}_{\underbrace{}_{0.4 \pi_{0}}} \\
& {\left[\tilde{L}_{n}, D_{\left\{m_{i}, \tilde{m}_{j}\right\}}\right]_{\mathrm{PB}}=\underbrace{\left[\tilde{L}_{n}, a_{m_{1}}^{\mu_{1}} \cdots a_{m_{r}}^{\mu_{r}}\right]_{\mathrm{PB}}}_{\text {2.47 }_{0}} \tilde{a}_{\tilde{n}_{1}}^{\nu_{1}} \cdots a_{\tilde{m}_{s}}^{\nu_{s}} e^{i N \frac{2 T}{k p} k x}+} \\
& +a_{m_{1}}^{\mu_{1}} \cdots a_{m_{r}}^{\mu_{r}} \underbrace{\left[L_{n}, \tilde{a}_{\tilde{n}_{1}}^{\nu_{1}} \cdots a_{\tilde{m}_{s}}^{\nu_{s}} e^{i N \frac{2 T}{k p} k x}\right]_{\mathrm{PB}}}_{2.45 \int_{0}} \text {. }
\end{aligned}
$$

This establishes the classical invariance of the DDF observables $D_{\left\{m_{i}, \tilde{m}_{j}\right\}}$. We next demonstrate how the Pohlmeyer invariants can be expressed in terms of DDF invariants.

\subsubsection{Expressing Pohlmeyer invartiants in terms of DDF invariants}

From the Fourier mode-like objects $A_{m}^{\mu}$ and $\tilde{A}_{m}^{\mu}$ one reobtains quasi-local fields ${ }^{1} \mathcal{P}_{ \pm}^{R}$ by an inverse Fourier transformation:

$$
\begin{aligned}
& \mathcal{P}_{-}^{R}(\sigma):=\frac{1}{\sqrt{2 \pi}} \sum_{m} A_{m}^{\mu} e^{+i m \sigma}=\left(\left(R_{-}\right)^{-1}\right)^{\prime}(\sigma) \mathcal{P}^{\mu}\left(\left(R_{-}\right)^{-1}(\sigma)\right) \\
& \mathcal{P}_{+}^{R}(\sigma):=\frac{1}{\sqrt{2 \pi}} \sum_{m} \tilde{A}_{m}^{\mu} e^{-i m \sigma}=\left(\left(R_{+}\right)^{-1}\right)^{\prime}(\sigma) \mathcal{P}^{\mu}\left(\left(R_{+}\right)^{-1}(\sigma)\right) .
\end{aligned}
$$

\footnotetext{
${ }^{1}$ It is interesting to discuss these fields, and in particular their quantization, from the point of view of worldsheet (quantum) gravity:

Clearly the $\mathcal{P}_{ \pm}^{\mu}(\sigma)$ are 'not physical' (do not Poisson commute with the constraints) because they evaluate the string's momentum and tension energy at a given value of the parameter $\sigma$, which of course has no physical relevance whatsoever. Heuristically, a physical observable may make recourse only to values of fields of the theory, not to values of auxiliary unphysical parameters. That is precisely the role played by the fields $R_{ \pm}$. They allow to characterize a point of the string purely in terms of physical fields (string oscillations). Instead of asking: "What is the value of $\mathcal{P}_{ \pm}$at $\sigma=3$ ?", we may ask the physically meaningful question: "What is the value of $\mathcal{P}_{ \pm}$at a point on the string where its configuration is such that $R_{+}=3$ ?" Quasi-local observables like the $\mathcal{P}_{ \pm}^{R}$, or rather their absence, are related to old and well known issues of (quantum) gravity in higher dimensions, often referred to in the context of "the problem of time" [13].

It is maybe instructive to note how these issues are resolved here for the worldsheet theory of the relativistic string, a toy example for quantum gravity when regarded as a theory of $1+1$ dimensional gravity. (Of course the string is rather more than a toy example for quantum gravity from the target space perspective.)
} 
This holds true on $\mathbf{P}_{k}(2.43)$ where we can use the fact that $R_{ \pm}$are invertible.

Comparison with $(2.20)$ shows that these are just reparameterizations of the original local worldsheet fields $\mathcal{P}_{ \pm}^{\mu}$, albeit with a reparameterization that varies from phase space point to phase space point, which is crucial for their invariance. But because the proof (2.23) of (2.22) involves only data available at a single point in phase space, it follows that for every invariant expression $F\left(\mathcal{P}_{-}\right)$of the worldsheet fields $\mathcal{P}_{-}^{\mu}$ with $\left[L_{m}, F\left(\mathcal{P}_{-}\right)\right]=0, \forall m$ we have

$$
F\left(\mathcal{P}_{-}\right)=F\left(\mathcal{P}_{-}^{R}\right)
$$

(on $\mathbf{P}_{k}$ ), and analogously for $\mathcal{P}_{+}$.

In summary we therefore obtain the following result:

On the restricted phase space $\mathbf{P}_{\mathrm{L}}(2.43)$ the classical Pohlmeyer invariants (2.16) can be expressed in terms of the classical DDF invariants (2.44) and the relation is

$$
Z^{\mu_{1} \cdots \mu_{N}}(\mathcal{P})=Z^{\mu_{1} \cdots \mu_{N}}\left(\mathcal{P}^{R}\right)
$$

where $\mathcal{P}$ is the ordinary worldsheet field $(2.13)$, and $\mathcal{P}^{R}$ is the linear combination (2.51) of classical DDF observables.

This can be expressed in words also as follows: The Pohlmeyer invariants are left intact when replacing oscillators by respective DDF observables in their oscillator expansion $\left(\alpha_{m}^{\mu} \rightarrow A_{m}^{\mu}, \tilde{\alpha}_{m}^{\mu} \rightarrow \tilde{A}_{m}^{\mu}\right)$. Note that the Pohlmeyer invariants are all of level 0 in the sense of (2.49) so that the level matching condition is trivially satisfied.

Because every polynomial in the DDF observables is consistently quantized by replacing $A_{m}^{\mu}$ and $\tilde{A}_{m}^{\mu}$ by the respective operators discussed in $\$ 2.1$ (p.4), this yields a consistent quantization of the Pohlmeyer invariants.

Finally, by simply generalizing the DDF invariants to the superstring, equation (2.53) defines the generalization of the Pohlmeyer invariants to the superstring. This is discussed in the next subsection:

\subsubsection{DDF and Pohlmeyer invariants for superstring}

The additional fermionc fields on the classical superstring shall here be denoted by $\Gamma_{ \pm}^{\mu}(\sigma)$, which are taken to be normalized so that their fermionic Poisson bracket reads

$$
\begin{aligned}
& \left\{\Gamma_{ \pm}^{\mu}(\sigma), \Gamma_{ \pm}^{\nu}(\kappa)\right\}_{\mathrm{PB}}= \pm 2 \eta^{\mu \nu} \delta(\sigma-\kappa) \\
& \left\{\Gamma_{ \pm}^{\mu}(\sigma), \Gamma_{\mp}^{\nu}(\kappa)\right\}_{\mathrm{PB}}=0 .
\end{aligned}
$$

The modes are of course

$$
\begin{aligned}
& b_{r}^{\mu}:=\frac{i}{\sqrt{4 \pi}} \int e^{-i r \sigma} \Gamma_{-}^{\mu}(\sigma) \\
& \tilde{b}_{r}^{\mu}=\frac{1}{\sqrt{4 \pi}} \int e^{+i r \sigma} \Gamma_{+}^{\mu}(\sigma)
\end{aligned}
$$


with non-vanishing brackets

$$
\begin{aligned}
\left\{b_{r}^{\mu}, b_{s}^{\nu}\right\}_{\mathrm{PB}} & =-i \eta^{\mu \nu} \delta_{r+s, 0} \\
\left\{\tilde{b}_{r}^{\mu}, \tilde{b}_{s}^{\nu}\right\}_{\mathrm{PB}} & =-i \eta^{\mu \nu} \delta_{r+s, 0}
\end{aligned}
$$

and the fermionic part of the super Virasoro constraints are

$$
\begin{aligned}
G_{r} & :=\frac{i}{\sqrt{2}} \int e^{-i r \sigma} \Gamma_{-}(\sigma) \cdot \mathcal{P}_{-}(\sigma) d \sigma=\sum_{m=-\infty}^{\infty} b_{r+m} \cdot \alpha_{-m} \\
\tilde{G}_{r} & :=\frac{1}{\sqrt{2}} \int e^{+i r \sigma} \Gamma_{+}(\sigma) \cdot \mathcal{P}_{+}(\sigma) d \sigma=\sum_{m=-\infty}^{\infty} \tilde{b}_{r+m} \cdot \tilde{\alpha}_{-m} .
\end{aligned}
$$

The point is that we can entirely follow the constructions discussed in 82.1 (p.4) to get classical DDF invariants $A_{m}^{\mu}$ and $B_{m}^{\mu}$ which Poisson-commute with the full set of super Virasoro constraints. For instance in the $\mathrm{R}$ sector the DDF observable $A_{m}^{\mu}$ is

$$
\begin{aligned}
A_{m}^{\mu} & :=\left\{G_{0}, \frac{i}{\sqrt{4 \pi}} \int \Gamma_{-}^{\mu}(\sigma) e^{-i m R_{-}(\sigma)}\right\} \\
& =\frac{1}{\sqrt{2 \pi}} \int d \sigma\left(\mathcal{P}_{-}^{\mu}(\sigma)+i m \frac{2 \pi \sqrt{2 T}}{k \cdot p} \Gamma_{-}^{\mu}(\sigma) k \cdot \Gamma_{-}(\sigma)\right) e^{-i m R_{-}(\sigma)} .
\end{aligned}
$$

By making the replacement $\alpha_{m}^{\mu} \rightarrow A_{m}^{\mu}$ in the ordinary Pohlmeyer invariant $Z^{\mu_{1} \cdots \mu_{N}}\left(\mathcal{P}_{-}\right)$ one obtains an object whose purely bosonic terms exactly coincide with the ordinary bosonic Pohlmeyer invariant and which furthermore has fermionic terms such that it superPoisson-commutes with all super Virasoro constraints. This object is therefore obviously the superstring generalization of the ordinary Pohlmeyer invariant of the bosonic string.

\section{Summary and Conclusion}

It has been shown that and how the Pohlmeyer invariants of the closed bosonic classical string can be expressed in terms of the classical analogs of the well known DDF operators. Heuristically, the construction is based on the observation that the DDF invariants are nothing but 'dynamically' reparameterized worldsheet oscillators and that the Pohlmeyer charges, being invariant under reparameterizations, remain unaffected under an exchange of ordinary oscillators with the respective DDF invariants.

This observation has some immediate consequences for the quantization program associated with the study of Pohlmeyer-invariants: A quantization of the DDF invariants in the usual way, leading to the DDF operators, is, by the above result, also a quantization of the Pohlmeyer invariants and their algebra. In particular this quantization is consistent in the sense that the commutator of two quantized Pohlmeyer invariants is itself again an invariant, simply because the algebra of DDF operators closes. This result should hence help to clarify some questions of the Pohlmeyer program which have so far remained open [2, 6]. 
But above that the understanding of the relation between DDF and Pohlmeyer invariants allows to immediately generalize the latter to the superstring and indeed to all two-dimensional superconformal field theories for which DDF operators can be written down. This in particular includes (super)strings on backgrounds which allow a free field realization of the worldsheet fields, such as (super)strings on pp-wave backgrounds [14].

From the point of view that the Pohlmeyer invariants are already included in the algebra of the common and well-known DDF observables one may wonder if they deserve any special attention at all.

In this respect we noted that the relation of the Pohlmeyer invariants to Wilson loops of constant large- $N$ gauge connections along the string is intriguingly reminiscent of the way states of string are expressed in terms of similar Wilson lines in the IIB(IKKT) matrix model. Maybe this points to an interesting relationship yet to be understood.

Another interesting aspect of the Pohlmeyer invariants is that they are all necessarily of vanishing oscillator level, quite contrary to the generic DDF invariant, and that they still form a complete set of invariant observables [4 in the sense that the knowlege of their classical values allows to locally reconstruct the string's worldsheet.

The vanishing of the level number of the Pohlmeyer invariants has the maybe interesting consequence that for the superstring they are linear combinations of terms of the form $\left[G_{-\nu}, c_{1}\right]\left[G_{-\nu}, c_{2}\right] \cdots\left[G_{-\nu}, c_{p}\right]$, (where $\nu=1 / 2$ in the NS and $\nu=0$ in the R sector and similarly for the other chirality sector), without any correction terms containing the coordinate field 0-modes (cf. (2.48).) Since $G_{-\nu}$ is a Dirac operator (the Dirac-Ramond operator) on loop space, this is essentially an exact differential form in the sense of Connes' noncommutative (spectral) geometry (e.g. [15]). Related observations have been made in section 4.4 of [16] and might point to an interesting meaning of the super-Pohlmeyer invariants, which has not fully emerged yet.

Note: After this work was completed we learned of the old articles [17, 18] where essentially the same results as given here are already reported. Their relevance for the Pohlmeyer program and for attempts at "alternative" quantizations of the string seems not to have been widely familiar [19.

\section{Acknowledgments}

I am grateful to Robert Graham for his support and to K.-H. Rehren for detailed discussions. I would also like to acknowledge interesting discussions with H. Nicolai and D. Bahns. Finally many thanks to A. P. Isaev for making me aware of his work.

This work has been supported by the SFB/TR 12 . 


\section{References}

[1] K. Pohlmeyer and M. Trunk, The invariant charges of the Nambu-Goto theory: Quantization of non-additive composition laws, Int. J. Mod. Phys. D 19 (2004) 115-148, hep-th/0206061.

[2] C. Meusburger and K. H. Rehren, Algebraic quantization of the closed bosonic string, Commun. Math. Phys. 237 (2003) 69-85 (2002). math-ph/0202041.

[3] K. Pohlmeyer, The Nambu-Goto theory of closed bosonic strings moving in 1+3-dimensional Minkowski space: The quantum algebra of observables, Ann. Phys. (NY) 8 (1999) 19-50. hep-th/9805057.

[4] K. Pohlmeyer and K.-H. Rehren, The invariant charges of the Nambu-Goto theory: Their geometric origin and their completeness, Commun. Math. Phys. 114 (1988) 177.

[5] T. Thiemann, The LQG-string: Loop quantum gravity quantization of String Theory I. Flat target space, hep-th/0401172.

[6] D. Bahns, The invariant charges of the Nambu-Goto string and canonical quantization, . hep-th/0403108.

[7] E. D. Giudice, P. D. Vecchia, and A. Fubini Ann. Phys. (NY) $\mathbf{7 0}$ (1972) 37\%.

[8] U. Schreiber, Pohlmeyer invariants are expressible in terms of DDF invariants, math-ph/0404007 (2004).

[9] M. Green, J. Schwarz, and E. Witten, Superstring Theory. Cambridge University Press, 1987.

[10] J. Polchinski, String Theory. Cambridge University Press, 1998.

[11] H. Aoki, S. Iso, H. Kawai, Y. Kitazawa, T. Tada, and A. Tsuchiya, IIB Matrix Model, Prog. Theor. Phys. Suppl. 134 (1999) 47-83. hep-th/9908038.

[12] K.-H. Rehren, 2004. (private communication).

[13] S. Carlip, Quantum gravity: A progress report, Rept. Prog. Phys. 64 (2001) 885. hep-th/0108040.

[14] Y. Hikida, Superstrings on NSNS pp-waves and their CFT duals, . hep-th/0303222.

[15] J. Varilli, An introduction to noncommutative geometry, . physics/9709045.

[16] U. Schreiber, On deformations of 2d SCFTs, J. High Energy Phys. 06 (2004) 058. hep-th/0401175.

[17] V. Borodulin and A. Isaev, On the infinite set of integrals of motion for a closed supersymmetric string, Phys. Lett. B 117 (1982) 69.

[18] A. Isaev, Integrals of the motion of a closed relativistic string, Theor. Math. Phys. 54 (1983) 134.

[19] U. Schreiber, 2004. weblog note http://golem.ph.utexas.edu/string/archives/000434.htm. 